\title{
A nationally quasi-experimental study to assess the impact of partial organized breast and cervical cancer screening programme on participation and inequalities
}

Heling Bao ${ }^{1}$, Limin Wang ${ }^{1}$, Matthew Brown², Mei Zhang ${ }^{1}$, Katherine Hunt ${ }^{3}$, Jiangli Di ${ }^{4}$, Zhenping Zhao', Shu Cong ${ }^{1}$, Jing Fan ${ }^{1}$, Liwen Fang ${ }^{1}$ and Linhong Wang ${ }^{1 *}$ (D)

\begin{abstract}
Background: Organized breast and cervical cancer screening programme could only provide services at no cost for a fraction of socioeconomic-deprived women in China and other low-resource countries, however, little evidence exists for whether such a programme effectively affect the participation and inequalities at the population level.

Methods: We used individual-level data from a nationally cross-sectional survey in 2014-2015 for breast and cervical cancer screening participation. Data for intervention and comparison grouping were from 2009 to 2014 national breast and cervical cancer screening programme, and counties covered by the programme were divided into intervention group. We assessed the impact of the intervention on the participation rates and the magnitude of inequalities with two approaches: multivariable multilevel logistic regressions adjusting for individual- and regionlevel covariates; and a difference analysis combined with propensity score matching that estimated the average intervention effect.

Results: Of 69,875 included women aged 35-64 years, 21,620 were classified into the intervention group and 43, 669 into the comparison group for breast cancer screening; and 31,794 into the intervention group, and 33,682 into the comparison group for cervical cancer screening. Participation rate was higher in intervention group than comparison group for breast cancer screening (25.3, 95\% confidential interval [CI], 22.8-27.7\%, vs 19.1, 17.4-20.7\%), and cervical cancer screening (25.7, 23.8-27.7\%, vs 21.5, 19.6-23.5\%), respectively. Intervention significantly increased the likelihood of participation for both breast cancer and cervical cancer screening in overall women, rural women and urban women, whereas the effect was significantly higher in rural women than urban women. The average intervention effect on the participation rate was an increase of 7.5\% (6.7-8.2\%) for breast cancer screening, and $6.8 \%(6.1-7.5 \%)$ for cervical cancer screening, respectively. The inequalities were significantly decreased by $37-41 \%$ (Continued on next page)
\end{abstract}

\footnotetext{
*Correspondence: linhong@chinawch.org.cn

${ }^{1}$ National Center for Chronic and Non-communicable Disease Control and Prevention, Chinese Center for Disease Control and Prevention, 27 Nanwei Road, Xicheng District, Beijing 100050, China

Full list of author information is available at the end of the article
} 


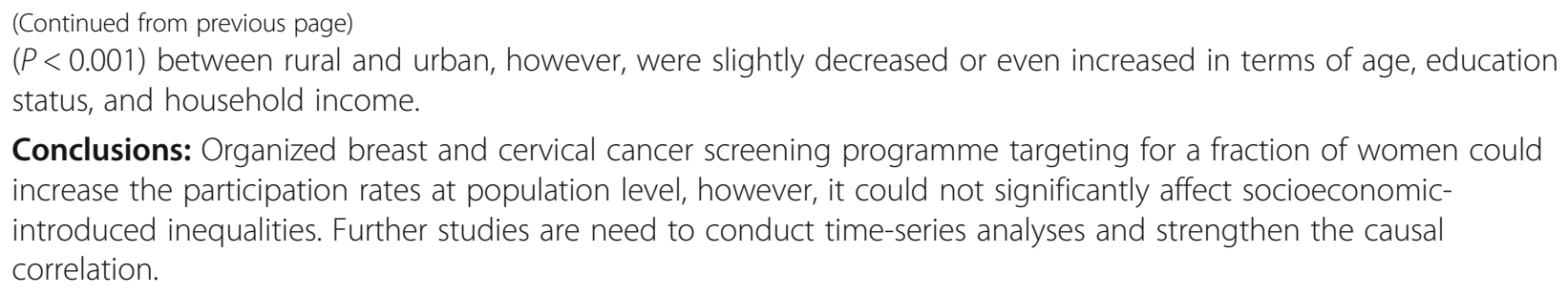

Keywords: Breast cancer screening, Cervical cancer screening, Participation, Inequality, Impact, Quasi-experimental study

\section{Background}

Breast cancer and cervical cancer are important contributors to the female cancer worldwide $[1,2]$. Screening for breast cancer could direct early detection and treatment, and reduce the death [3], whereas cervical cancer screening could detect cervical precancerous lesions, stop the occurrence of invasive cancer, and further decrease the cervical cancer incidence [4]. Nonetheless, morbidity and mortality of breast and cervical cancer decrease less or remain unchanged in low-resource settings and lead to widening disparities [5].

Population-based organized breast or cervical cancer screening programme with universal coverage could effectively increase the participation [6, 7]. In low-resource settings, organized programme could only benefit a fraction of women who were difficult to access to services, such as socioeconomic-deprived women [8-10], and therefore would not gain equivalent coverage as that in developed countries. Previous studies showed that the removal of out-of-pocket costs for breast and cervical cancer screening could increase the participation rate [10], but increase the magnitude of inequalities in terms of demographic or socioeconomic status [11]. However, the impact of such a programme for a fraction of eligible women on the participation rates and inequalities is less studied. The absence of evidence is a major obstacle to the implementation and evaluation of such programme in low-resource settings.

The incidence of breast and cervical cancer are substantial in China [2]. However, there was no organized screening programme for breast cancer before 2008 [12]; and cervical cancer screening programme was once conducted at the workplace $[13,14]$ but broke down following the reforms of the health-care system in the early 1980s [15]. It was estimated that the participation rate for breast or cervical cancer screening was less than 30\% in Chinese women aged 20 years or older [16]. In 2009, the Chinese government initiated a national breast and cervical cancer screening programme for women living in rural areas [14]. Because there were many age-eligible women in rural China, the government budget could only afford cost-removing screening for a fraction of women in the programme counties. Specifically, the programme selected counties across China and recruited a fraction of rural women aged 35-64 years. Thus, the initiate of the programme allowed for a comparison of participation rates between women who were in programme counties and those who were not in, and that between rural and urban areas.

In the study, we used a nationally representative, quasi-experimental study to assessed how the organized breast and cervical cancer screening programme for a fraction of eligible women affected the participation rates and socioeconomic inequalities at population level. Furthermore, we estimated the average intervention effect attributed to the programme.

\section{Methods \\ Study design and participant}

We used the individual-level data from the 2014-2015 Chinese chronic disease and risk factor survey to measure the participation rates of breast and cervical cancer screening among women aged 35-64 years. We designed a quasi-experimental study by combining the nationally representative survey with the data from the 2009-2014 breast and cervical cancer screening programme. Sampled counties in the survey were divided into intervention group and comparison group with no randomization, according to whether they were covered by the programme. Women in the survey were categorized as rural women (target) and urban women (non-target) based on the place of residence. We compared the difference in screening participation rates between intervention group and control group for overall, rural women, and urban women, respectively. Multivariable multilevel regressions were used to assess the impact of the programme on the participation rates for breast and cervical cancer screening. Difference analyses combined with propensity score matching were used to estimate the average intervention effect. Propensity score matching is increasingly used to balance the bias of covariates in the evaluation of intervention when randomization is not feasible in observational studies [17]. Additionally, we analyzed the change of the relative and 
absolute inequalities in terms of demographic and socioeconomic status.

This study was approved by the ethics committees of the National Center for Chronic and Non-Communicable Disease Control and Prevention. All participants provided written informed consent before any study procedures.

\section{Procedure}

During 2009-2014, the organized screening programme covered 714 counties for breast cancer screening and 1306 counties for cervical cancer screening across China. County maternal and child health care center in the programme enrolled a fraction of rural women aged 3564 years (the age range was 30-59 years during 20092011 and then switched to 35-64 years since 2012), and provided them with breast cancer screening, or cervical cancer screening, or both at no cost. According to the National Statistics Census [18], rural areas were defined as villages and townships in a county, whereas urban areas were defined as towns, suburbs, or central areas. Additionally, women who were not included in the organized programme could also access to self-supporting screening in health check-up or clinic visiting. During 2009-2014, the programme screened 4.8 million rural women for breast cancer, and screened 40 million for cervical cancer nationwide.

The national cross-sectional survey was described in greater detail previously [19]. Briefly, the survey applied a multistage, systematic, clustered sampling in which 297 counties were randomly selected to create a nationally representative sample. Participants $(n=179,347)$ aged 18 years or older were enrolled, and characteristics of demo-graphic, risk factors, or behaviors related to chronic diseases were collected through a face-to-face interview. All 69,875 female participants aged 35-64 years were abstracted for this study. Considering the birth cohort effect, those women were $30-59$ years in 2009 which was also eligible for the programme.

We linked the 297 sampled counties in the survey with the counties in the programme by unique county code and name. The sampled counties overlapped with the programme counties were classified into intervention group, whereas other counties were classified into comparison group. For breast cancer screening, 99 sampled counties were in the intervention group and 198 were in the comparison group; for cervical cancer screening, 142 were in the intervention group and 155 were in the comparison group. There were 90 sampled counties covered by both breast and cervical cancer screening programme (see Additional file 1 Fig. S1). Women in the survey were also categorized as rural women or urban women according to the definition concordant with the programme. Rural women in the intervention group were eligible for cost-removing screening; by contrast, urban women in the intervention group and all women in the comparison group were ineligible in the programme.

\section{Outcome and covariate measurement}

The primary outcomes were participation rates of breast and cervical cancer screening. Women who reported receiving breast or cervical cancer screening at least once during 2009-2014 were defined as screening participation. Participation rate was calculated from the number of women participated in screening divided by all women.

Individual-level covariates in the study included: age group; nationality (Han/others); marital status (never/ married/others); education attainment (primary school and lower/junior/senior or higher); employment status (not working/ non-agricultural employment/agriculture employment); medical insurance status (no insurance/insurance for employed resident/insurance for unemployed resident); household income (separated by quartiles and don't know); health checkup (less than 1 year/every 2-3 years/more than 3 years); self-rated health (good or very good/fair/bad or very bad). We also combined the data from national statistics census to collected county-level covariates, including: the proportion of residents residing in urban areas (urbanization), the proportion of residents $\geq 25$ years who are college graduates (education status), the number of health worker per 1000 residents (health care), and the per capita gross domestic product (GDP). County-level variables were categorized into tertiles.

\section{Statistical analysis}

The participation rates were estimated for breast and cervical cancer screening, respectively, and 95\% confidence intervals (CI) were estimated accounting for complex survey design. Rao-scott $\chi^{2}$ test was used to compare the difference for categorical variables. We estimated the participation rates for rural and urban women in intervention group or comparison group, respectively, and compared them to show the direct and indirect effect introduced by the programme. We used multivariable multilevel logistic regressions with random intercepts at county- and province- levels, adjusting for individual- and county-level covariates, to estimate the effect of the intervention on the likelihood of participation. In these models, we added intervention term alone, intervention term restricting to rural women, and an interaction term of intervention and residency in rural, respectively, to show different effect of the intervention for overall, rural, and urban women, respectively.

Individual matching is an alternative approach to improve balance of covariates in observational studies, especially when the sample size is large enough [17]. In 
this study, individuals in the intervention group were matched in a 1:1 ratio to the comparison group based on propensity scores, which was calculated from logistic regressions including individual covariates. We conducted the matching for each subgroup of interest using greedy matching method [20]. Then, we calculated the average intervention effects using methods as described by Farzadfar and colleagues [21]. Briefly, we calculated the differences in participation rates between the intervention group and comparison group on the balanced dataset, taking account of the subclassification of residency in place, age group, household income, and education attainment.

Relative index of inequality (RII) was the ratio between the estimated participation rate among women with the highest level (e.g., age, income, or education) and the lowest level, whereas slope index of inequality (SII) measured the absolute difference between the highest level and lowest level [22]. We estimated RII and SII in terms of rural-urban, age, household income, and education by use of generalized linear models, and used an interaction term of socioeconomic variable and intervention to test the significance.

All analyses were done separately for breast and cervical cancer screening. Probability values for statistical tests were two tailed with $P<0.05$ as statistically significant. Multilevel logistic regressions were estimated with MLwiN (version 2.30), and other analyses were done with SAS software (version 9.4).

\section{Results}

Of 69,875 included women in the survey, 65,289 were included in the analysis for breast cancer screening and 65 , 476 women were included for cervical cancer screening.

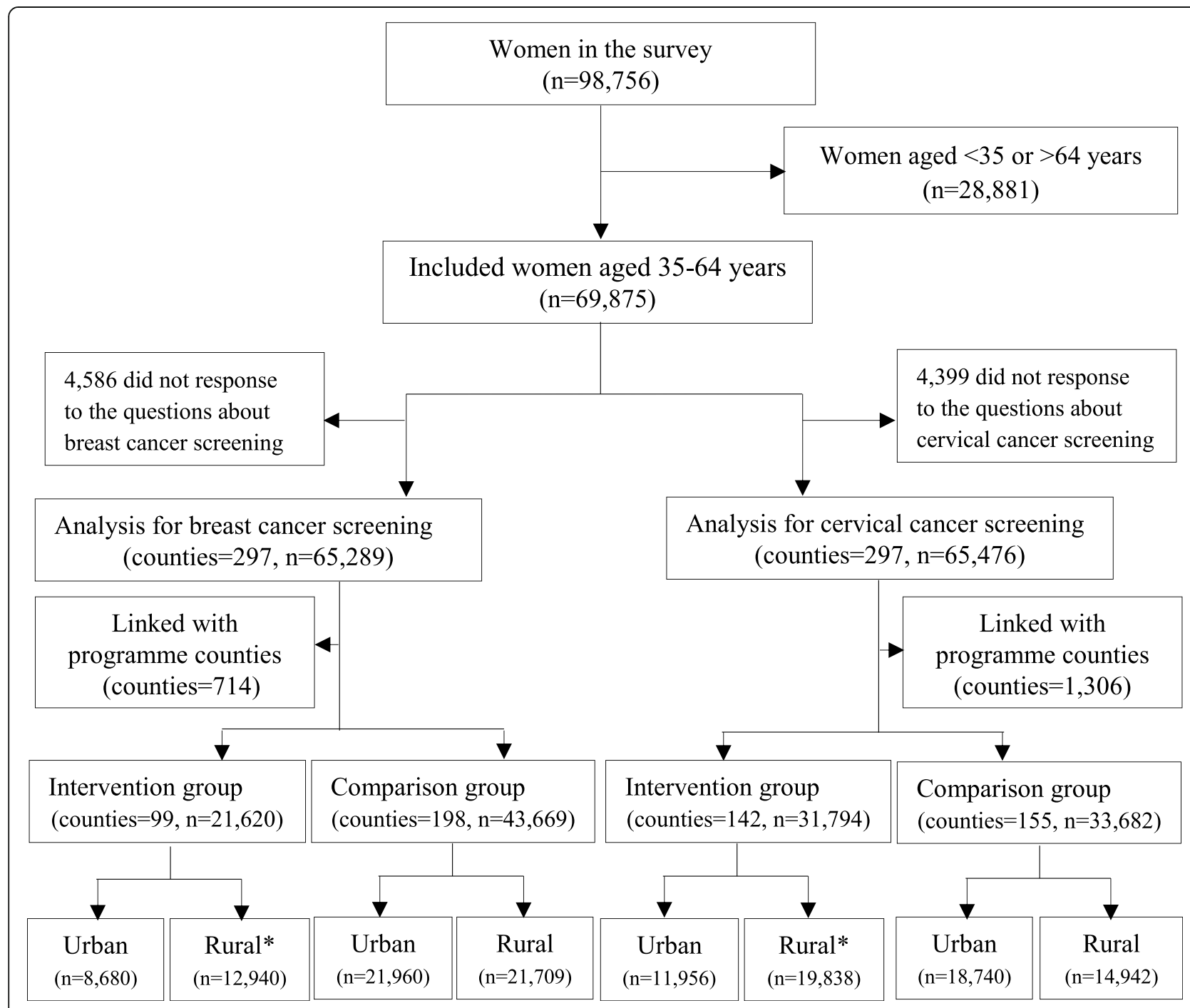

Fig. 1 Flowchart of eligible participants and grouping in the study. Notes: ${ }^{*}$ In these rural areas, the programme provided cost-removal breast and cervical cancer screening to women aged 35-64 years 
Table 1 The breast and cervical cancer screening participation rates in intervention and comparison groups by characteristics

\begin{tabular}{|c|c|c|c|c|c|c|}
\hline & \multicolumn{3}{|c|}{ Breast cancer screening } & \multicolumn{3}{|c|}{ Cervical cancer screening } \\
\hline & $\overline{n(\%)}$ & $\begin{array}{l}\text { Intervention } \\
\%(95 \% \mathrm{Cl})\end{array}$ & $\begin{array}{l}\text { Comparison } \\
\%(95 \% \mathrm{Cl})\end{array}$ & $\mathrm{n}(\%)$ & $\begin{array}{l}\text { Intervention } \\
\%(95 \% \mathrm{Cl})\end{array}$ & $\begin{array}{l}\text { Comparison } \\
\%(95 \% \mathrm{Cl})\end{array}$ \\
\hline Overall & $65,289(100.0)$ & $25.3(22.8-27.7)$ & $19.1(17.4-20.7)$ & $65,476(100.0)$ & $25.7(23.8-27.7)$ & $21.5(19.6-23.5)$ \\
\hline \multicolumn{7}{|l|}{ Place of residence } \\
\hline Rural areas & $34,649(53.1)$ & $23.2(19.9-26.4)$ & $12.9(11.0-14.8)$ & $34,780(53.1)$ & $25.7(23.5-27.9)$ & $16.9(14.6-19.3)$ \\
\hline Urban areas & $30,640(46.9)$ & $28.4(25.8-31.0)$ & $25.2(23.2-27.1)$ & $30,696(46.9)$ & $30.8(28.3-33.3)$ & $28.9(26.6-31.1)$ \\
\hline \multicolumn{7}{|l|}{ Age group } \\
\hline $35-39$ & $6941(10.6)$ & $29.6(26.7-32.5)$ & $24.0(21.5-26.5)$ & $6963(10.6)$ & $31.8(29.5-34.1)$ & $27.7(24.9-30.4)$ \\
\hline $40-44$ & $10,819(16.6)$ & $33.8(30.5-37.0)$ & $24.1(21.9-26.3)$ & $10,837(16.6)$ & $35.0(32.3-37.6)$ & $28.1(25.6-30.7)$ \\
\hline $45-49$ & $13,373(20.5)$ & $31.9(28.8-34.9)$ & $22.7(20.7-24.7)$ & $13,439(20.5)$ & $33.1(30.6-35.6)$ & $26.4(23.9-29.0)$ \\
\hline $50-54$ & $11,536(17.7)$ & $29.8(26.9-32.8)$ & $22.3(20.3-24.3)$ & $11,570(17.7)$ & $29.4(27.1-31.7)$ & $24.5(22.2-26.7)$ \\
\hline $55-59$ & $12,425(19.0)$ & $21.6(19.2-24.1)$ & $17.5(15.6-19.4)$ & $12,468(19.0)$ & $21.0(19.1-22.9)$ & $20.9(18.6-23.1)$ \\
\hline $60-64$ & $10,195(15.6)$ & $14.1(12.3-16.0)$ & $14.1(12.3-15.9)$ & $10,199(15.6)$ & $14.5(12.9-16.1)$ & $15.2(13.3-17.2)$ \\
\hline \multicolumn{7}{|l|}{ Education attainment } \\
\hline Primary school and lower & $12,481(19.1)$ & $16.9(13.8-20.0)$ & $9.0(7.1-10.9)$ & $12,569(19.2)$ & $18.3(16.1-20.4)$ & $11.7(9.4-13.9)$ \\
\hline Junior school & $41,724(63.9)$ & $26.7(24.2-29.3)$ & $18.7(16.9-20.4)$ & $41,783(63.8)$ & $28.0(25.9-30.1)$ & $21.2(19.1-23.3)$ \\
\hline Senior school and higher & $11,047(16.9)$ & $44.4(41.1-47.7)$ & $38.2(35.6-40.8)$ & $11,088(16.9)$ & $44.1(41.2-47.0)$ & $38.7(35.7-41.8)$ \\
\hline \multicolumn{7}{|l|}{ Annual household income } \\
\hline 1st (lowest) quartile & $13,010(20.0)$ & $20.9(19.5-23.2)$ & $13.6(11.5-15.7)$ & $13,062(20.0)$ & $23.4(21.3-25.6)$ & $15.8(13.5-18.0)$ \\
\hline 2nd quartile & $13,837(21.2)$ & $27.9(24.8-31.1)$ & $19.2(17.2-21.3)$ & $13,918(21.3)$ & $29.0(26.5-31.5)$ & $21.8(19.4-24.2)$ \\
\hline 3th quartile & $12,076(18.5)$ & $32.4(28.5-36.3)$ & $23.4(21.2-25.6)$ & $12,122(18.6)$ & $31.9(28.7-35.2)$ & $25.8(23.4-28.3)$ \\
\hline 4th (highest) quartile & $11,416(17.5)$ & $36.9(32.7-41.1)$ & $30.5(27.2-33.8)$ & $11,381(17.4)$ & $35.7(31.8-39.7)$ & $33.6(30.0-37.2)$ \\
\hline Refused/don't know & $14,820(22.7)$ & $20.4(18.1-22.8)$ & $17.6(15.8-19.5)$ & $14,863(22.7)$ & $22.2(20.2-24.1)$ & $20.0(17.9-22.1)$ \\
\hline
\end{tabular}

Note: $95 \% \mathrm{Cl}$ was estimated by Taylor series variances estimation approach accounting for complex sampling design

Abbreviations: Cl Confidential interval

For breast cancer screening, 21,620 women were divided into the intervention group and 43,669 were into the comparison group; for cervical cancer screening, 31,794 women were divided into the intervention group and 33, 682 were into the comparison group (Fig. 1). There were significant differences in the distribution of some sociodemographic characteristics between the intervention and comparison groups (see Additional file 1 Table S1).

Table 1 pt?>showed that participation rates of breast and cervical cancer screening in the intervention group were significantly higher than the comparison group $(P<0.001$ for both). Participation rates of the intervention group were significantly higher than the comparison group in rural women, however, the differences were not significant in urban women. Figure 2 showed the agespecific participation rates of rural women in the intervention group were significantly higher than rural women in the comparison group, whereas there were not similar patterns for urban women between intervention and comparison group. Additionally, participation rates in women aged 40-54 years, lower education level, and lower household income in the intervention group were significantly higher than the corresponding women in the comparison group (see Additional file 1 Table S2).

Table 2 showed the intervention significantly increased the likelihood of participation for breast cancer screening $(\mathrm{OR}=1.63,95 \% \mathrm{CI} 1.56-1.71)$ and cervical cancer screening $(\mathrm{OR}=1.74,95 \% \mathrm{CI} 1.38-2.20)$. The interaction term showed the intervention had significantly higher effect in rural women than in urban women $(P<0.05$ for both); nonetheless, the intervention effect was also significant in urban women. The combination intervention of breast and cervical cancer in the programme had significantly higher effect for either breast cancer or cervical cancer screening than separate intervention. The lower likelihood of screening participation rates was significantly associated with demographic or socioeconomic status in terms of older, lower education attainment, and lower household income (see Additional file 1 Table S3).

After matching, the distribution of main demographic factors was not significantly different between the two groups (see Additional file 1 Table S4). The postmatching difference analyses showed that, intervention increased participation rate of breast cancer screening by 
A

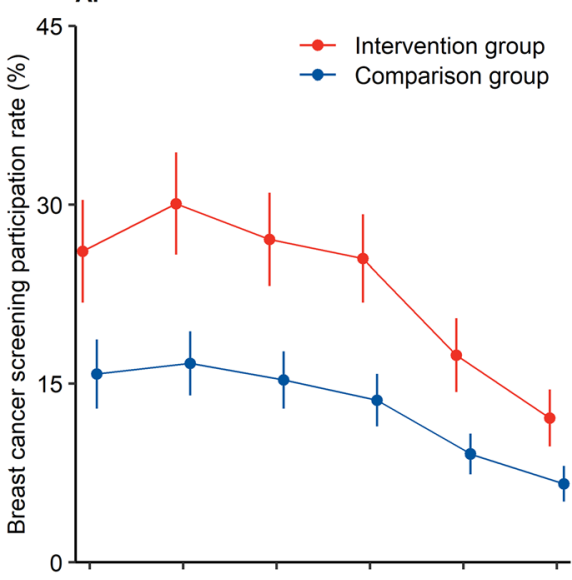

C.

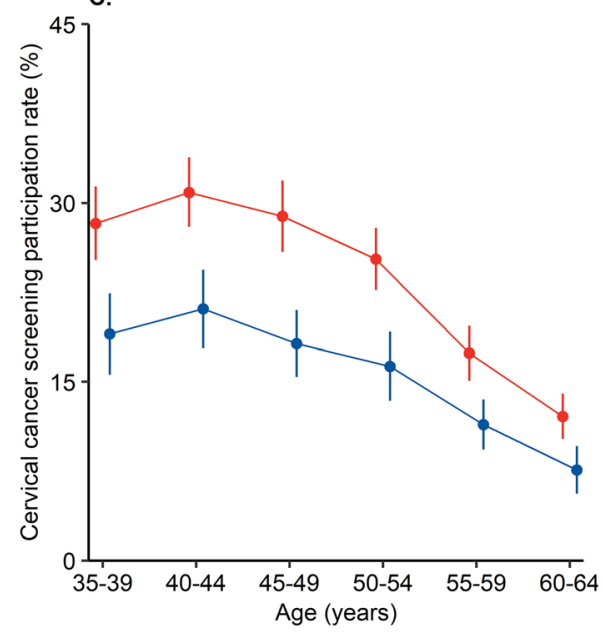

B.

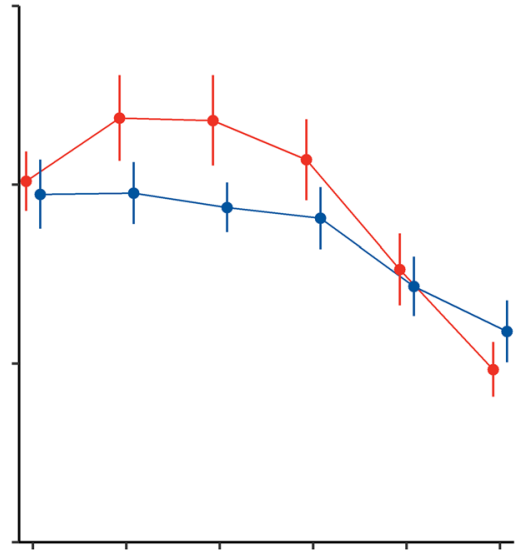

D.

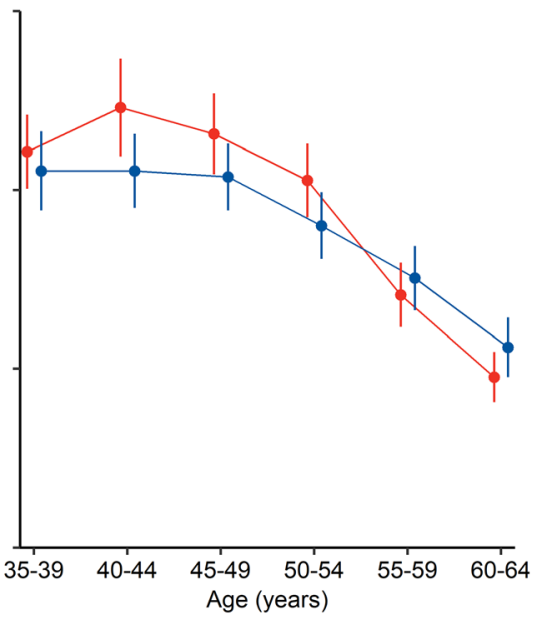

Fig. 2 The age-specific participation rates of breast and cervical screening in the intervention and comparison groups by place of residence. a breast cancer screening participation rates in rural women. b breast cancer screening participation rates in urban women. c cervical cancer screening participation rates in rural women. $\mathbf{d}$ cervical cancer screening participation rates in urban women. Note: $95 \% \mathrm{Cl}$ was estimated by Taylor series variances estimation approach accounting for complex sampling design

7.5\% (95\%CI 6.7-8.2\%), and cervical cancer screening by 6.8\% (95\%CI 6.1-7.5\%) (Fig. 3 and Additional file 1 Table S5). Consistent with findings of intervention effect by multilevel regressions, the average intervention effect was higher in rural women compared with urban women. By contrast, the average intervention effects were relatively lower in older, lower household income, and lower education level subgroups. (see Additional file 1 Table S6).

Table 3 showed that intervention substantially decreased the magnitude of inequalities on participation rates between urban and rural women for both breast and cervical cancer screening, and the relative and absolute indicators significantly decreased ranging from $37.1 \%$ to $-41.9 \%$. Although the relative inequality indicator in terms of household income and education attainment had significantly decreased, absolute indicators changed with no significance. By contrast, relative and absolute inequality indicators in terms of age group substantially increased ranging from 23.1 to $76.9 \%$.

\section{Discussion}

In the nationally representative analysis, the organized programme targeting for a fraction of rural women significantly increased the participation rates of breast and cervical screening for not only rural women but also urban women. The average intervention effects in participation rates of breast and cervical cancer screening was $7.5 \%$ for breast cancer screening and $6.8 \%$ for cervical cancer screening, respectively. The intervention effect was significantly higher in rural women than urban women. Using the results of post-matching estimates according to age, the absolute total number of women 
Table 2 Results of multivariable multilevel logistic regressions for the intervention effect

\begin{tabular}{|c|c|c|c|c|}
\hline & \multicolumn{2}{|l|}{ Breast cancer screening } & \multicolumn{2}{|c|}{ Cervical cancer screening } \\
\hline & Adjusted OR (95\%Cl) & $P$ value & Adjusted OR (95\%Cl) & $P$ value \\
\hline \multicolumn{5}{|l|}{ Model $1 \mathrm{a}^{*}$} \\
\hline Intervention vs. comparison & $1.63(1.56-1.71)$ & $<0.001$ & $1.74(1.38-2.20)$ & $<0.001$ \\
\hline \multicolumn{5}{|l|}{ Model $2^{b^{*}}$} \\
\hline Intervention vs. comparison (rural areas) & $1.77(1.40-2.26)$ & $<0.001$ & $1.84(1.45-2.33)$ & $<0.001$ \\
\hline \multicolumn{5}{|l|}{ Model $3^{c^{*}}$} \\
\hline Intervention vs. comparison (urban areas) & $1.54(1.18-2.00)$ & 0.002 & $1.63(1.29-2.07)$ & $<0.001$ \\
\hline Intervention interaction with rural areas & $1.31(1.17-1.47)$ & $<0.001$ & $1.13(1.01-1.25)$ & 0.036 \\
\hline \multicolumn{5}{|l|}{ Model $4^{\mathrm{d}^{*}}$} \\
\hline Intervention for one cancer vs. comparison & $1.20(1.07-1.36)$ & 0.002 & $1.52(1.12-2.06)$ & 0.007 \\
\hline Intervention for both cancer vs. comparison & $1.69(1.61-1.77)$ & $<0.001$ & $1.90(1.46-2.47)$ & $<0.001$ \\
\hline \multicolumn{5}{|c|}{$\begin{array}{l}\text { Abbreviations: OR Odds ratio, CI Confidential interval } \\
{ }^{*} \text { All models were adjusting for covariates, including: individual-level age, education attainment, household income, employment status, health insurance, health } \\
\text { checkup, self-rated health, and region-level per capita gross domestic product, education status, urbanization, and density of health care worker, with random } \\
\text { effect in levels of county and province. All the covariates in the model were assessed using variance influence factor, tolerance, and characteristic root to } \\
\text { avoid collinearity } \\
\text { Note: }{ }^{\mathrm{a}} \text { Including intervention alone } \\
{ }^{b} \text { Including the crossed classification of intervention and residency in rural, and then comparing the rural women in the intervention group to the rural women in } \\
\text { the comparison } \\
\text { c Including the interaction term of intervention and residence. Intervention term showed the intervention effect in urban women; the interaction term of } \\
\text { intervention and residency in rural showed the differential effect of intervention in rural women compared with that in urban women } \\
\text { d Intervention group was further divided into intervention for one cancer screening alone and for both breast and cervical cancer screening }\end{array}$} \\
\hline
\end{tabular}

aged 35-64 years who participated in breast and cervical cancer screening as a result of the programme during 2009-2014 was estimated to be 21.9 million and 20.4 million women, respectively. For breast cancer, the detected cases in early stage due to increase in intervention was estimated as 15,987 according to detection rate of breast cancer at 0-3 stage [23]. Based on the detection rate of cervical intraepithelial lesions grade $2 / 3$ or adenocarcinoma in situ [24], the detected cases with cervical precancerous lesions was estimated as 23,063 cases, who had higher risk of progression to invasive cancer.

The present study showed that participation rates of urban women who were ineligible for the cost-removing screening in the intervention group also significantly increased during the period. This finding indicates the indirect effect of the organized programme on the ineligible women, consistent with previous studies $[10,11]$. Yutaka et al. found that free-screening for women with targeted age might positively affect the screening rates of ageineligible women, however, they did not give the estimation of the indirect effect [10]. In our study, we conducted the analyses restricting to urban women (ineligible for cost-removing screening) in the intervention group or comparison group, to estimate the indirect effect. Individual matching balanced the distribution of characteristics that may affected the screening. The reason behind the indirect effect would be explained as raising awareness, providing education, addressing barriers, motivating women, and peer pressure from the programme [10, 25]. Furthermore, the implementation of the programme based on the maternal and child health care network may also help to remove structural, financial, and technical barriers and improve the availability and accessibility of cancer prevention in programme counties [26, 27].

On the assumption that free-screening (4.8 million breast cancer screening and 40 million cervical cancer screening) were delivered to unscreened women, the average intervention effect should be substantially higher for cervical cancer compared with that for breast cancer screening. However, the average intervention effect was relatively high for breast cancer screening in our results. Many reasons may explain this. Firstly, the proportion of counties implementing the two programme is higher in breast cancer screening programme than that in cervical cancer screening programme (91\% vs $63 \%$ ), and therefore the combination of two screening programme has higher effect on the breast cancer screening compared with cervical cancer screening. Secondly, for cervical cancer, women with better compliance behavior, cervical and/or vaginal symptoms, or history of human papillomavirus infection or abnormal cytology were more likely to attend the programme at a shorter interval. The magnitude of over-screening for cervical cancer needs to be further explored. Thirdly, the outreach modalities might differently affect the participation rate of breast and cervical cancer screening, e.g., group education and small media might be more effective in participation of breast cancer screening than cervical cancer screening [28, 29]. Finally, the questionnaire was not designed for the evaluation, and several screening modalities for breast cancer, such as breast self- 


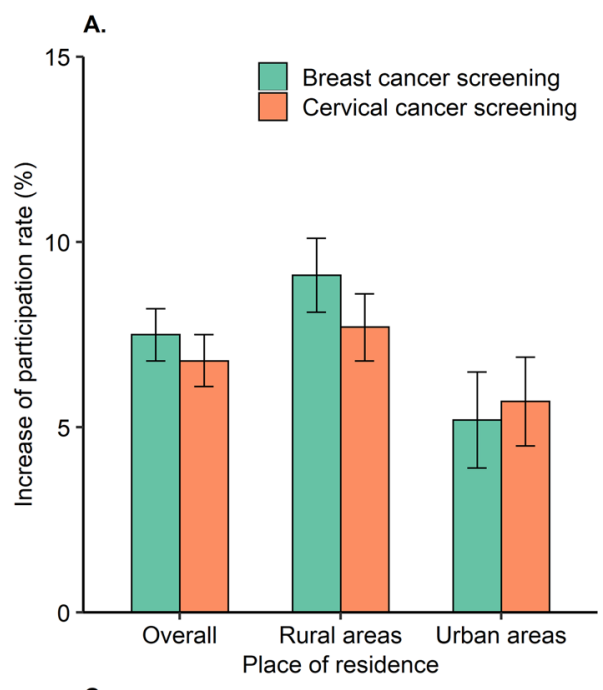

C.

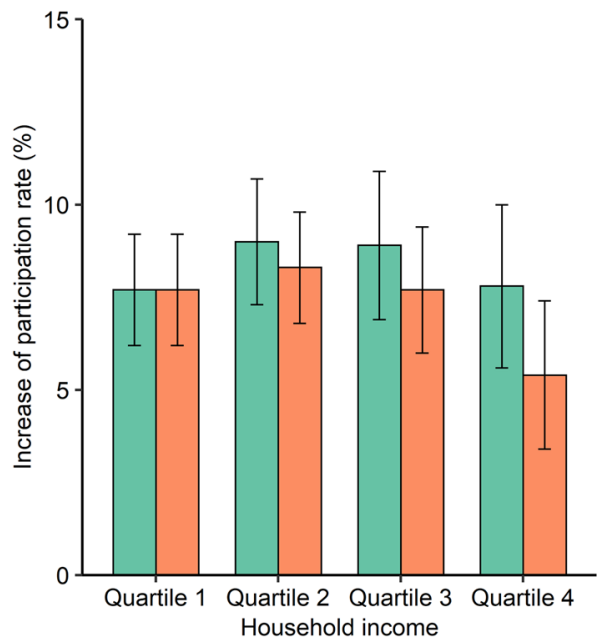

B.

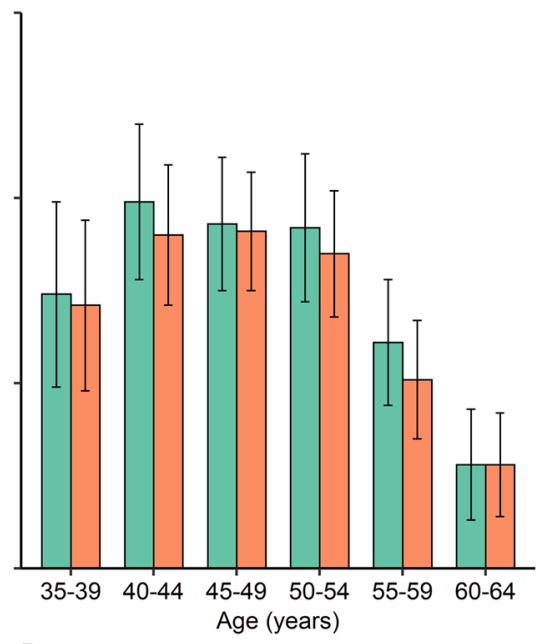

D.

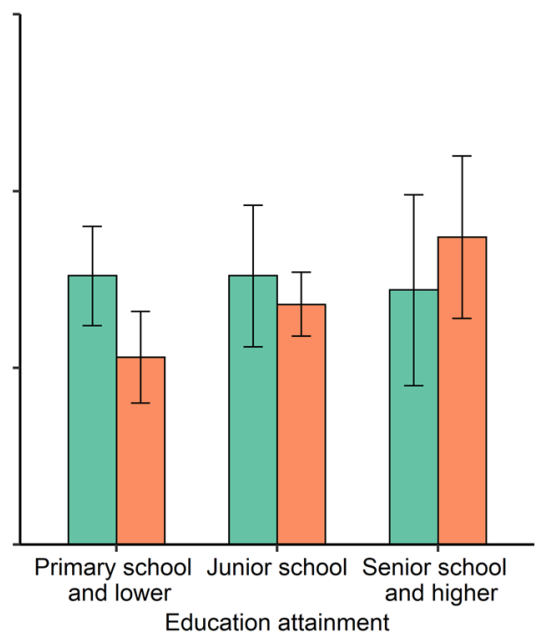

Fig. 3 The average intervention effect on the participation rates of breast and cervical cancer screening. a intervention effect in overall, rural, and urban women, respectively. $\mathbf{b}$ intervention effect stratified by age group. $\mathbf{c}$ intervention effect stratified by household income. $\mathbf{d}$ intervention effect stratified by education attainment. Note: The average intervention effect was calculated from the difference of participation rates between intervention and comparison group on subclassifications combined with propensity score matching

examination, might be included in individuals' responses [11]. Therefore, the average intervention effect may be widened for breast cancer screening but be narrowed for cervical cancer screening.

Our results showed that an organized screening programme targeting for a fraction of women would affect the magnitude of inequalities on screening participation, consistent with previous studies [30, 31]. Intervention targeting for rural women significantly narrowed the rural-urban inequalities, however, the magnitudes in terms of age, household income, and education attainment were slightly decreased or not. Although relative inequalities are decreased in some socioeconomic terms, the absolute indicators that is recommended as primacy show different patterns. Substantial increases in both relative and absolute indicators in terms of age group may decrease the cost-effectiveness of cancer screening, because the mortality and morbidity of breast and cervical cancer are higher among older women than younger $[1,2]$. Although intervention with removal of costs positively affected the participation, organized activities co-existing with unrestricted opportunistic screening might consume public resources $[11,31]$. That screening register system combining the organized programme with opportunistic screening in routine practice might play a role in reduction of the inequalities, but the effect should be further studied.

Due to the huge expenditure of population-based breast and cervical cancer screening, organized programme serving for a fraction of underserved women at no cost isfeasible in low-resource settings. Although our results show that such programme could positively affect the 
Table 3 Estimates and percent change of relative and absolute inequality indicators for cervical and breast cancer screening participation

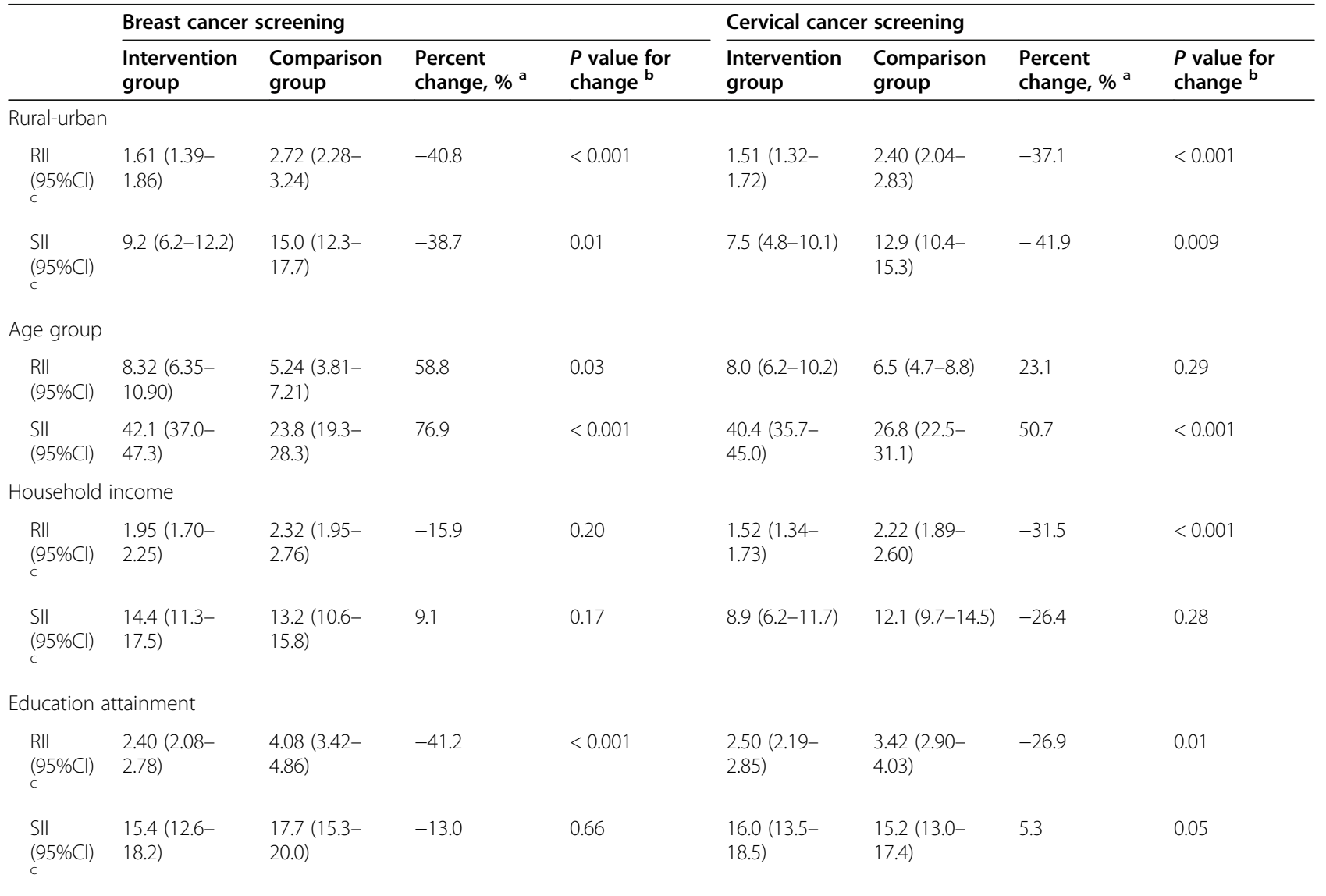

Abbreviations: RII Relative index of inequalities, SII Slope index of inequalities

${ }^{a}$ Percent change was calculated from the difference between intervention and comparison divided by the comparison

${ }^{b} P$ values were calculated from generalized linear model including the interaction term of intervention and indicators

c These indicators were calculated adjusting for age group

population-level participation rate for cancer screening, it also has several challenges [32-35]. According to Zhao and et al. [36], if the elimination of cervical cancer would be achieved in China, the coverage of once-in-a-lifetime cervical cancer screening is required to reach $90 \%$ in urban and $33 \%$ in rural with vaccination of $95 \%$ coverage for girls aged 12 years. It demands the programme a larger government-support budget to expanded the screening coverage from the current status in rural and urban areas [19]. Moreover, the cost-effectiveness of the programme should be considered as priority in low-resource settings [37]. Inefficient delivery strategy would widen the disparities within women who were in lower socioeconomic position and at higher risk of developing cancer [38]. Serving women who were rarely or never screened should be a key component [39, 40]. To address these barriers, improved delivery strategies are urgently needed to expand the programme moving forwards, such as timely individual invitation [40, 41], monitoring and management of screening performance [42], and innovation to develop the evidence base for actions [43, 44].

To our knowledge, this is the first study to use nationally representative, cross-sectional study to evaluate the impact of an organized breast and cervical cancer programme serving for a fraction of underserved women on the participation and inequalities in low-resource settings. Large sample size and generalized conclusions are our strengths, which include multilevel data sources, and enable a robust statistical analysis and control for potentially confounding variables.

Some limitations should be discussed. A key limitation is that our results might be affected by selection bias, because no randomization could control for unmeasured or unknown confounders. Although propensity score matching was used to maximum decreased the bias caused by the different distribution of demographic factors, some unmeasured factors would affect the results which should be interpreted with caution. A round of 
cross-sectional survey may not consider the dynamic changes of programme and other covariates associated with screening participation, and hence, time-series analyses should be conducted to strengthen the causal correlation. Self-reported information may introduce the misclassification bias. Nonetheless, two distinct statistical approaches were applied to control for potential confounding in different ways, and the conclusions about the impact of the intervention were consistent. Finally, the survey could not distinguish whether the selfreported testing was performed for cancer screening or disease diagnosis, but the post-matching estimate for intervention would be not affected because the bias would distributed equally between the intervention and comparison group.

\section{Conclusions}

An organized breast and cervical cancer screening programme targeting for a fraction of rural women could significantly increase the participation rates for both programme-eligible and -ineligible women, and reduce the rural-urban inequalities, however, the magnitude of inequalities in terms of age, household income, and education were slightly decreased or not. The causal correlation of such programme and change of participation rate at the population level needs further time-series study.

\section{Supplementary Information}

The online version contains supplementary material available at https://doi. org/10.1186/s12885-020-07686-4.

Additional file 1. Supplementary data.

\section{Abbreviations \\ Cl: Confidence intervals; RII: Relative index of inequalities; SII: Slope index of inequalities}

\section{Acknowledgements}

We would like to thank all the research staff from local Centers for Disease Control and Prevention for their collection of data in the cross-sectional survey.

\section{Authors' contributions}

BHL: Conceptualization, formal analysis, visualization, writing original draft, and editing. WLH: Conceptualization, supervision, project administration, and review. WLM, ZM and ZZP: Project administration, investigation, data curation. MB and KH: Visualization, review and editing. FLW, DJL, CS and FJ: Data curation, investigation, review and editing. WLH had full access to all the data in the study and take responsibility for the integrity of the data and the accuracy of the data analysis. All authors have read and approved the final version of manuscript.

\section{Funding}

This study was supported by grants from the Chinese Ministry of Science and Technology (National Key R\&D Program of China: 2016YFC0901300, 2016YFC0901301). None of the funding organizations were involved in the design and implementation of the study; collection, management, analysis, and interpretation of the data; preparation, review, or approval of the manuscript; or the decision to submit the manuscript for publication.

\section{Availability of data and materials}

The dataset used and/or analyzed during the current study are available from corresponding author on reasonable request.

\section{Ethics approval and consent to participate}

The study protocol was approved by the ethical review committee of the National Center for Chronic and Non-communicable Disease Control and Prevention, China CDC. Written informed consent was obtained from all study participants.

\section{Consent for publication}

Not applicable.

\section{Competing interests}

The authors have declared that they have no conflicts of interests.

\section{Author details}

${ }^{1}$ National Center for Chronic and Non-communicable Disease Control and Prevention, Chinese Center for Disease Control and Prevention, 27 Nanwei Road, Xicheng District, Beijing 100050, China. ${ }^{2}$ Center for Global Health, China Office, National Cancer Institute, National Institutes of Health, Bethesda, USA. ${ }^{3}$ Susan G. Komen, 5005 LBJ Freeway, Suite 526, Dallas, TX 75244, USA. ${ }^{4}$ National Center for Women and Children's Health, Chinese Center for Disease Control and Prevention, 12 Dahuisi Road, Haidian District, Beijing 100081, China.

Received: 12 March 2020 Accepted: 24 November 2020

Published online: 04 December 2020

\section{References}

1. Global Burden of Disease Cancer C, Fitzmaurice C, Dicker D, Pain A, Hamavid H, Moradi-Lakeh M, MF MI, Allen C, Hansen G. Woodbrook R et al. The Global Burden of Cancer 2013. JAMA Oncol. 2015;1:505-27.

2. Bray F, Ferlay J, Soerjomataram I, Siegel RL, Torre LA, Jemal A. Global cancer statistics 2018: GLOBOCAN estimates of incidence and mortality worldwide for 36 cancers in 185 countries. CA Cancer J Clin. 2018;68:394-424.

3. Lauby-Secretan B, Scoccianti C, Loomis D, Benbrahim-Tallaa L, Bouvard V, Bianchini F, Straif K. Breast-cancer screening--viewpoint of the IARC working group. N Engl J Med. 2015;372:2353-8.

4. Volerman A, Cifu AS. Cervical cancer screening. JAMA. 2014;312:2279-80.

5. Arnold M, Renteria E, Conway DI, Bray F, Van Ourti T, Soerjomataram I. Inequalities in cancer incidence and mortality across medium to highly developed countries in the twenty-first century. Cancer Causes Control. 2016;27:999-1007.

6. Adams EK, Breen N, Joski PJ. Impact of the National Breast and cervical Cancer early detection program on mammography and pap test utilization among white, Hispanic, and African American women: 1996-2000. Cancer. 2007:109:348-58.

7. Bucchi L, Baldacchini F, Mancini S, et al. Estimating the impact of an organised screening programme on cervical cancer incidence: a 26-year study from northern Italy. Int J Cancer. 2019;144:1017-26.

8. Van Dyne EA, Hallowell BD, Saraiya M, et al. Establishing baseline cervical Cancer screening coverage - India, 2015-2016. MMWR Morb Mortal Wkly Rep. 2019;68(1):14-9.

9. Arrossi S, Paolino M, Laudi R, et al. Programmatic human papillomavirus testing in cervical cancer prevention in the Jujuy demonstration project in Argentina: a population-based, before-and-after retrospective cohort study. Lancet Glob Health. 2019;7:e772-e83.

10. Ueda Y, Sobue T, Morimoto A, et al. Evaluation of a free-coupon program for cervical cancer screening among the young: a nationally funded program conducted by a local government in Japan. J Epidemiol. 2015;25: 50-6.

11. Tabuchi T, Hoshino T, Nakayama T, Ito Y, loka A, Miyashiro I, Tsukuma H. Does removal of out-of-pocket costs for cervical and breast cancer screening work? A quasi-experimental study to evaluate the impact on attendance, attendance inequality and average cost per uptake of a Japanese government intervention. Int J Cancer. 2013;133:972-83.

12. Fan LF, Kathrin SW, Li JJ, Jessica SL, Diamne MF, Yu KD, Chen WQ, Saho ZM, Paul EG. Breast cancer in China. Lancet Oncol. 2014;15:70567-9. 
13. Tao N, Lu HZ, Shen Y, Chen WZ. The research on the cervical cancer prevention and treatment in the past 23-years. Shanghai Med. 1984;7:33842.

14. Di J, Rutherford S, Chu C. Review of the cervical Cancer burden and population-based cervical Cancer screening in China. Asian Pac J Cancer Prev. 2015;16:7401-7.

15. Zhang D, Unschuld PU. China's barefoot doctor: past, present, and future. Lancet. 2008;372:1865-7.

16. Wang B, He M, Wang L, Engelgau MM, Zhao W, Wang L. Breast Cancer screening among adult women in China, 2010. Prev Chronic Dis. 2013;10: 130-6.

17. Austin PC. An introduction to propensity score methods for reducing the effects of confounding in observational studies. Multivar Behav Res. 2011;46: 399-424.

18. National Bureau of Statistics. National Statistics Census 2014. Available at: http://www.stats.gov.cn/. Accessed at May 20, 2019.

19. Bao H, Zhang L, Wang L, Zhang M, Zhao Z, Fang L, Cong S, Wang L. Significant variations in the cervical cancer screening rate in China by individul-level and geographical measures of socioeconomic status: a multilevel model analysis of a nationally representative survey dataset. Cancer Med. 2018;7:2089-100.

20. Lori S. Parsons ORG, Seattle, WA. Reducing Bias in a Propensity Score Matched-Pair Sample Using Greedy Matching Techniques 2001; doi: https:// doi.org/10.1016/j.micinf.2011.07.011.Innate.

21. Farzadfar F, Murray CJ, Gakidou E, et al. Effectiveness of diabetes and hypertension management by rural primary health-care workers (Behvarz workers) in Iran: a nationally representative observational study. Lancet. 2012;379:47-54.

22. Mackenbach JP, Stirbu I, Roskam AJ, et al. Socioeconomic inequalities in health in 22 European countries. N Engl J Med. 2008;358:2468-81.

23. Ma L, Ren WH, Zhao YX, et al. Cost-effectiveness of optimized ultrasoundbased breast cancer screening for Chinese rural women in 2015. Chin Cancer. 2019;28:891-5.

24. Luo XM, Song $L$, Wu JL, et al. Analysis of the reported data of national rural cervical cancer screening project from 2012 to 2013. Chin J Prev Med. 2016; 50:346-50.

25. Levano W, Miller JW, Leonard B, Bellick L, Crane BE, Kennedy SK, Haslage NM, Hammond W, Tharpe FS. Public education and targeted outreach to underserved women through the National Breast and cervical Cancer early detection program. Cancer. 2014;16:2591-6.

26. Lee NC, Wong FL, Jamison PM, Jones SF, Galaska L, Brady KT, Wethers B, Stokes-Townsend GA. Implementation of the National Breast and cervical Cancer early detection program: the beginning. Cancer. 2014;120:2540-8.

27. Benard VB, Royalty J, Saraiya M, Rockwell T, Helsel W. The effectiveness of targeting never or rarely screened women in a national cervical cancer screening program for underserved women. Cancer Causes Control. 2015; 26:713-9.

28. Sabatino SA, Lawrence B, Elder R, Mercer SL, Wilson KM, DeVinney B, Melillo S, Carvalho M, Taplin S, Bastani R, et al. Effectiveness of interventions to increase screening for breast, cervical, and colorectal cancers: nine updated systematic reviews for the guide to community preventive services. Am J Prev Med. 2012;43:97-118.

29. Peitzmeier SM, Khullar K, Potter J. Effectiveness of four outreach modalities to patients overdue for cervical cancer screening in the primary care setting: a randomized trial. Cancer Causes Control. 2016:27:1081-91.

30. Moser K, Patnick J, Beral V. Inequalities in reported use of breast and cervical screening in Great Britain: analysis of cross sectional survey data. BMJ. 2009; 338:b2025.

31. Palencia L, Espelt A, Rodriguez-Sanz M, et al. Socio-economic inequalities in breast and cervical cancer screening practices in Europe: influence of the type of screening program. Int J Epidemiol. 2010;39:757-65.

32. Strasser-Weippl K, Chavarri-Guerra Y, Villarreal-Garza C, Bychkovsky BL, Debiasi M, Liedke PE, Soto-Perez-de-Celis E, Dizon D, Cazap E, de Lima Lopes G Jr, et al. Progress and remaining challenges for cancer control in Latin America and the Caribbean. Lancet Oncol. 2015;16:1405-38.

33. Lantz PM, Mullen J. The National Breast and cervical Cancer early detection program: 25 years of public health service to low-income women. Cancer Causes Control. 2015;26:653-6.

34. Arbyn M, Rebolj M, De Kok IM, Fender M, Becker N, O'Reilly M, Andrae B. The challenges of organising cervical screening programmes in the 15 old member states of the European Union. Eur J Cancer. 2009;45:2671-8.
35. Brown ML, Klabunde CN, Cronin KA, White MC, Richardson LC, McNeel TS Challenges in meeting healthy people 2020 objectives for cancer-related preventive services, National Health Interview Survey, 2008 and 2010. Prev Chronic Dis. 2014;11:E29.

36. Xia CF, Hu SY, Xu XQ, et al. Projections upt ot 2100 and a budget optimisation strategy towards cervical cancer elimination in China: a modelling study. Lancet Public Health. 2019;4:3462-72.

37. Lewin S, Lavis JN, Oxman AD, et al. Supporting the delivery of cost-effective interventions in primary health-care systems in low-income and middleincome countries: an overview of systematic reviews. Lancet. 2008;372:92839.

38. Simard EP, Fedewa S, Ma J, Siegel R, Jemal A. Widening socioeconomic disparities in cervical cancer mortality among women in 26 states, 19932007. Cancer. 2012;118:5110-6.

39. White MC, Wong FL. Preventing premature deaths from breast and cervical cancer among underserved women in the United States: insights gained from a national cancer screening. Cancer Causes Control. 2015;26:805-9.

40. Frieden TR. Six components necessary for effective public health program implementation. Am J Public Health. 2014;104:17-22.

41. Yancy B, Royalty JE, Marroulis S, Mattingly C, Benard VB, DeGroff A. Using data to effectively manage a national screening program. Cancer. 2014;16: 2575-83.

42. DeGroff A, Royalty JE, Howe W, Buckman DW, Gardner J, Poister T, Hayes N. When performance management works: a study of the National Breast and cervical. Cancer. 2014;16:2566-74.

43. Plescia M, White MC. The National Prevention Strategy and breast cancer screening: scientific evidence for public health action. Am J Public Health. 2013;103:1545-8.

44. DeGroff A, Cheung K, Dawkins-Lyn N, Hall MA, Melillo S, Glover-Kudon R. Identifying promising practices for evaluation: the National Breast and cervical Cancer early detection program. Cancer Causes Control. 2015;26: $767-74$

\section{Publisher's Note}

Springer Nature remains neutral with regard to jurisdictional claims in published maps and institutional affiliations.

Ready to submit your research? Choose BMC and benefit from:

- fast, convenient online submission

- thorough peer review by experienced researchers in your field

- rapid publication on acceptance

- support for research data, including large and complex data types

- gold Open Access which fosters wider collaboration and increased citations

- maximum visibility for your research: over $100 \mathrm{M}$ website views per year

At BMC, research is always in progress.

Learn more biomedcentral.com/submissions 